\title{
Prediction of preeclampsia in the first trimester of pregnancy using maternal characteristics, mean arterial pressure, and uterine artery Doppler data in a Brazilian population
}

\author{
Juliana de Freitas Leite, Guilherme Antonio Rago Lobo, Paulo Martin Nowak, Irene Reali Antunes, \\ Edward Araujo Júnior, David Baptista da Silva Pares \\ Department of Obstetrics, Paulista School of Medicine, Federal University of São Paulo (EPM-UNIFESP), São Paulo, SP, Brazil
}

\section{Objective}

To evaluate the performance of the preeclampsia (PE) screening algorithm of the Fetal Medicine Foundation (FMF) during the first trimester in a Brazilian population using maternal characteristics, mean arterial pressure (MAP), and uterine artery Doppler data.

\section{Methods}

This is a prospective cohort study that evaluated 701 pregnant women during the first trimester ultrasound screening for chromosomal abnormalities (11-13+6 weeks). All patients provided information regarding clinical and obstetric history, MAP, and mean uterine artery pulsatility index (mean PI). Patients were assigned to four groups based on the presence of PE and gestational age at delivery: group 1 (control), patients without hypertensive disorders $(n=571)$; group 2, PE and delivery before 34 weeks of gestation ( $n=7)$; group 3, PE and delivery before 37 weeks of gestation, including patients from group 2 and patients that presented PE with delivery between 34 and 37 weeks $(n=17)$; and group 4, PE and delivery before 42 weeks of gestation, including patients from both groups 2 and 3 and patients that presented PE with delivery between 37 and 42 weeks of gestation $(n=34)$.

\section{Results}

After the exclusion of 96 patients, we evaluated the data of 605 patients. By combining maternal characteristics, MAP, and the mean uterine artery PI for the detection of PE, we found a sensitivity of $71.4 \%$ in group $2,50 \%$ in group 3 , and $41.2 \%$ in group 4 (false positive rate $=10 \%$ ).

\section{Conclusion}

Using maternal characteristics, MAP, and uterine artery Doppler data, we were able to identify a significant proportion of patients who developed preterm PE.

Keywords: Preeclampsia; Prediction; First trimester; Mean artery pressure; Uterine artery Doppler

\section{Introduction}

Preeclampsia (PE) affects about $2-4 \%$ of pregnancies and is the biggest cause of maternal and perinatal morbidity and mortality [1-4]. In Brazil, it is responsible for about a quarter of maternal deaths [5]. Severe hypertensive disorders were the main cause of severe maternal morbidity and the mortality index was $10.7 \%$ in a Brazilian multicenter study [6].

Besides the impact on mortality, PE is one of the main
Received: 2018.10.18. Revised: 2019.04.03. Accepted: 2019.04.17. Corresponding author: Edward Araujo Júnior Rua Belchior de Azevedo, 156 apto. 111 Torre Vitoria, São Paulo, SP CEP 05089-030, Brazil

E-mail: araujojred@terra.com.br

https://orcid.org/0000-0002-6145-2532

Articles published in Obstet Gynecol Sci are open-access, distributed under the terms of the Creative Commons Attribution Non-Commercial License (http://creativecommons. org/licenses/by-nc/3.0/) which permits unrestricted non-commercial use, distribution, and reproduction in any medium, provided the original work is properly cited.

Copyright $\odot 2019$ Korean Society of Obstetrics and Gynecology 


\section{Obstetrics \& Gynecology Science}

Vol. 62, No. 6, 2019

causes of severe maternal morbidity. This disease causes multisystem commitment due to a generalized vasospasm associated with endothelial lesions and a change in microcirculation at the level of the central nervous system, kidneys, lungs, liver, retina, and other organs. For this reason, it has the potential to cause multiple organ failure and sequelae.

Despite numerous studies in the last decades, the physiopathology of PE is not completely known. It has a multifactorial pathogenesis which involves immunological, genetic, hormonal, and environmental factors. Until now, the only cure for PE has been the removal of the placenta, which seems to be the pathogenic root of all of the disease's manifestations [7]. In PE, trophoblastic invasion is deficient and causes an increase in the resistance of uterine and placental circulation, placental hypoxia, and local oxidative stress [8]. This process leads to a systemic inflammatory response and vasospasms which result in hypertension, edema, and proteinuria.

Several studies have been conducted in an attempt to identify high risk PE patients in the first trimester of pregnancy so that they may benefit from an effective prophylaxis with acetylsalicylic acid (ASA) before 16 weeks [9]. In order to obtain a higher sensitivity in identifying these patients, the following variables have been combined: maternal characteristics, mean arterial pressure (MAP), uterine artery pulsatility index (PI), and biochemical markers.

The Fetal Medicine Foundation (FMF) has developed a prediction algorithm for PE in the first trimester. Since it uses all variables, it achieves expressive results in detecting preterm PE, with a sensitivity of $76 \%$ (false positive [FP], $10 \%$ ). Although the combined method has an acknowledged superiority, in developing countries such as Brazil biochemical markers are unavailable in the public health system and are thus unavailable for the majority of the population. Unfortunately, without these biomarkers, the sensitivity rate for identifying patients at high risk for PE is lower.

Thus, the goal of our study was to evaluate the performance of the FMF algorithm when predicting PE in the first trimester by using the most feasible variables in our context: maternal characteristics, MAP, and uterine artery Doppler data.

\section{Materials and methods}

We conducted a prospective cohort study during the first trimester screening in the Department of Obstetrics, Paulista School of Medicine- Federal University of São Paulo (EPMUNIFESP). The project was approved by the research ethics committee of the Federal University of São Paulo (UNIFESP) and all participants signed a consent form.

This study included the first trimester screening examination of 701 patients between 11 and 13+6 weeks of gestation. In addition to the complete morphological assessment, including the risk for trisomy, we performed a uterine artery Doppler velocimetry via the abdomen according to FMF recommendations. Patients were in the semirecumbent position and a sagital section of the uterus and internal cervical os was obtained by transabdominal ultrasound. After the internal cervical os was identified, the transducer was tilted from side to side and color Doppler was used to identify the uterine arteries as aliasing vessels coursing along the side of the cervix and uterus. Pulsed wave Doppler obtained three similar consecutive waveforms from the ascending branch of the uterine artery at the level of the internal cervical os. The $\mathrm{PI}$ was measured and the mean PI of the left and right arteries was calculated [10].

After the ultrasonography examination, we conducted a thorough anamnesis of patients which included information regarding personal and obstetrical history of chronic hypertension $(\mathrm{CH})$, diabetes mellitus (DM), systemic lupus erythematosus (SLE), antiphospholipid antibodies syndrome (AAS), in vitro fertilization, regular use of ASA, PE, parity and prematurity, and family history of PE.

Weight and height were also measured. Blood pressure was measured only once, after a minimum rest period of five minutes, with the patient seated and the cuff placed on the right or left arm which was maintained at heart level. For the establishment of diastolic blood pressure (DBP), we used the 5th Korotkoff sound, which corresponds to the disappearance of sound. A periodically calibrated manual mercury sphygmomanometer was used according to the sector's maintenance regulations.

With regard to maternal outcomes, information was collected by analyzing patient records. Pressure levels during admission and hospitalization, laboratory examinations, proteinuria values, usage and dosage of hypotensive medication, eventual usage of magnesium sulfate, and gestational age at hospitalization and delivery were all evaluated in order to characterize hypertensive disorders.

To identify PE cases, we followed the definition as stated in 


\section{Obstetrics \& Gynecology Science}

Juliana de Freitas Leite, et al. Prediction of preeclampsia

the guidelines of the National High Blood Pressure Education Program (NHBPEP) [7]: systolic blood pressure (SBP) higher or equal to $140 \mathrm{mmHg}$ and/or DBP higher or equal to $90 \mathrm{mmHg}$ on at least two occasions, with an interval between measurements of at least 4 hours in a previously normotensive patient, which is associated with a 24 hour proteinuria of $300 \mathrm{mg}$ or more, or a urine dipstick test with at least one cross, or, exceptionally, the presence of $1 \mathrm{~g}$ of protein in the isolated sample.

Patients were divided into five groups based on maternal outcome: group 1, control ( $n=571$ patients); group 2, PE and delivery before 34 weeks $(n=7)$; group 3, PE and delivery before 37 weeks $(n=18[7+11])$; group 4, PE and delivery before 42 weeks $(n=34[7+11+16])$, and group 5 , gestational hypertension $(n=12)$.

We therefore retrospectively calculated each patient's individual risk of developing PE before 34, 37, and 42 weeks of pregnancy, using the first trimester screening program software, version 2.8.0, based on the FMF 2012 specifications. This software calculates the risk of aneuploidies by combining maternal history, pregnancy characteristics, fetal nuchal translucency thickness and other ultrasound markers, and maternal serum biochemistry in the first trimester. The program also calculates separate risks for $\mathrm{PE}$, fetal growth restriction, and spontaneous preterm delivery based on maternal history and pregnancy characteristics, ultrasound, and biochemical and biophysical markers in the first trimester. In addition, it provides a detailed database of patients screened, a reporting system, and a detailed audit section.

The following variables were evaluated using multivariate logistic regression analysis: maternal characteristics, mean uterine artery $\mathrm{Pl}$, and MAP value (a single measurement was repeatedly filled in the four designated fields since the original program includes four arterial pressure measurements and considers their average).

Data were compiled in an Excel spreadsheet (Microsoft Corp., Redmond, WA, USA) and analyzed by SPSS software version 19.0 (SPSS Inc., Chicago, IL, USA). The maternal characteristics, MAP, and uterine artery Doppler data of patients in groups 2, 3, and 4 were compared to group 1 (control). The chi-square $\left(\chi^{2}\right)$ or Fisher's exact test was used to compare qualitative variables, and the Student's t-test was used to compare quantitative variables. The mean uterine artery PI was converted to multiples of median (MoMs) by FMF software in order to correct the influence of gestational age, race, weight, parity, and smoking [11]. After the FP rate was set at 5 and $10 \%$, we determined the cut-off, the calculation sensitivity and specificity, the positive and negative predictive values, and the positive and negative likelihood ratios for combined risk using the FMF algorithm. The predictive performance was also evaluated by the receiver operating characteristic (ROC) curve, with calculation of the area under the curve. The rejection level for the null hypothesis was set as $P \leq 0.05$ (5\%).

\section{Results}

A total of 84 patients out of 701 were excluded due to: loss to follow-up (48), unclassified hypertensive disorder (14), late miscarriage (11), fetus or newborn with chromosomal anomalies (4), fetus or newborn with severe structural defects (3), fetal death before the 24th gestational week (2), regular use of ASA (2), and gestational hypertension (12). Fig. 1 shows

701 women were evaluated at $11-13+6$ weeks
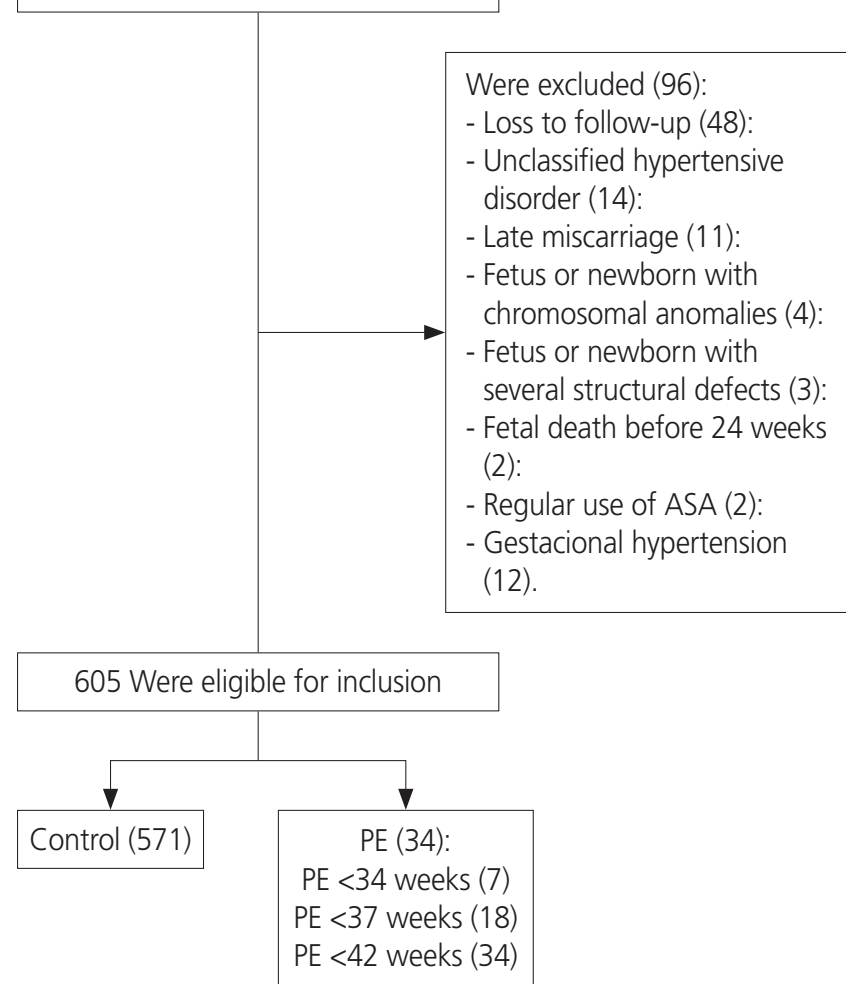

Fig. 1. Screening and follow up of the included patients on the study. ASA, acetylsalicylic acid; PE, preeclampsia. 


\title{
Obstetrics \& Gynecology Science
}

\author{
Vol. 62, No. 6, 2019
}

the flow chart of the patients included in the study.

Out of the 605 patients included in this study, 34 (5.6\%) developed PE. At any gestational age, the incidence of PE was $5.6 \%$ (34 cases). The average age was 29.8 years (standard deviation $[S D]=6.5$ years). A total of $309(54.3 \%)$ patients were classified as mixed race, $250(41.3 \%)$ were nulliparous, 49 had a history of PE ( $8 \%$ of the total sample and $23 \%$ among those who developed PE), 65 (10.7\%) were chronically hypertensive, $23(3.8 \%)$ were diabetic, $16(2.6 \%)$ had SLE or AAS, and 10 (1.6\%) had chronic renal disease. The median gestational age was 39 weeks (interquartile range $[I Q R]=38-40$ ) and the median birth weight was 3,190 grams (IQR=2,850-3,500). Tables 1 and 2 show the maternal characteristics of our sample.

The incidence of PE before 34 weeks was 1\% (7 cases). The development of the disease in this group was significantly associated with the presence of a history of $\mathrm{PE}, \mathrm{CH}$, and chronic renal disease. Other risk factors did not show significant differences. The mean uterine artery PI was significantly higher in the PE groups than in the control group. However, there were no significant differences in MAP values. The sensitivity achieved through the combination of variables (maternal characteristics, MAP, and mean uterine artery PI) was
$71.4 \%$, with an FP rate of $10 \%$ and a negative predictive value of $99.6 \%$.

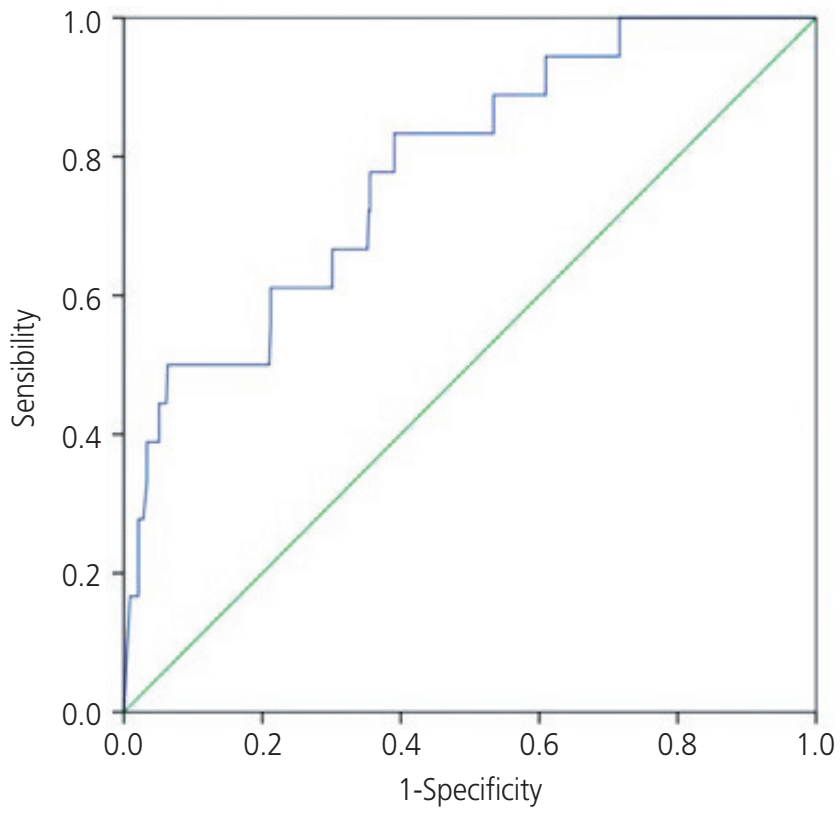

Fig. 2. Receiver operating characteristics curve for preeclampsia prediction in the first trimester using the combination of the following variables: maternal characteristics, mean artery pressure and uterine artery Doppler.

Table 1. Maternal variables evaluated in Brazilian patients $(n=605)$ in accordance with gestational age at delivery

\begin{tabular}{lcccc}
\hline Variables & $\begin{array}{c}\text { Control } \\
\text { Mean (SD) }\end{array}$ & $\begin{array}{c}\text { PE<34 weeks } \\
\text { Mean (SD) }\end{array}$ & $\begin{array}{c}\text { PE<37 weeks } \\
\text { Mean (SD) }\end{array}$ & $\begin{array}{c}\text { PE<42 weeks } \\
\text { Mean (SD) }\end{array}$ \\
\hline Age $(\mathrm{yr})$ & $29.8(6.5)$ & $33.1(8.2)$ & $29.3(7.1)$ & $29.9(6.9)$ \\
Weight $(\mathrm{kg})$ & $66.3(13.8)$ & $64.1(8.7)$ & $69.8(13.5)$ & $73.0(18.3)$ \\
Height $(\mathrm{cm})$ & $1.60(0.06)$ & $1.56(0.06)$ & $1.59(0.08)$ & $1.59(0.07)$ \\
BMl & $26(5.0)$ & $26.5(3.9)$ & $27.6(4.2)$ & $28.8(6.2)$ \\
MAP $(\mathrm{mmHg})$ & $79.6(8.9)$ & $86.1(13.5)$ & $88.2(13.7)$ & $88.5(13.0)$ \\
UtAPI & $1.49(0.5)$ & $1.90(0.45)$ & $1.78(0.45)$ & $1.75(0.48)$ \\
\hline
\end{tabular}

SD, standard deviation; PE, preeclampsia; BMI, body mass index; MAP, mean arterial pressure; UtAPI, uterine artery pulsatility index.

Table 2. Maternal medical history and characteristics in accordance with gestational age at delivery

\begin{tabular}{lcccc}
\hline Variables & Control (\%) & PE<34 weeks (\%) & PE<37 weeks (\%) & PE<42 weeks (\%) \\
\hline Nulliparity & 40.5 & 57.1 & 50.0 & 44.1 \\
Previous PE & 12.1 & 66.7 & 55.6 & 53.3 \\
CH & 8.8 & 71.4 & 38.9 & 44.1 \\
Diabetes mellitus & 3.3 & 0.0 & 5.6 & 11.8 \\
Thrombophilia & 2.5 & 14.3 & 11.1 & 5.9 \\
Smoke & 2.5 & 14.3 & 11.1 & 5.9 \\
\hline
\end{tabular}

PE, preeclampsia; CH, chronic hypertension; Thrombophilia, systemic lupus erythematosus or antiphospholipid syndrome. 


\section{Obstetrics \& Gynecology Science}

Juliana de Freitas Leite, et al. Prediction of preeclampsia

The incidence of PE before 37 weeks was 3\% (18 patients). The presence of a history of $\mathrm{PE}, \mathrm{CH}$, and chronic renal disease was significantly associated with the development of PE before 37 weeks. The MAP and mean PI values were significantly higher than in the control group.

The sensitivity of the combined method for detection of preterm PE was $50 \%$, the negative predictive value was $98.1 \%$, and the FP rate was $10 \%$. The performance of this method was evaluated by the area under the curve (0.78) and is represented in the ROC curve of Fig. 2.

The incidence of PE before 42 weeks was 5.6\% (34 cases). In this group, the development of the disease was significantly associated with the following variables: weight, body mass index, and a history of $\mathrm{PE}, \mathrm{CH}, \mathrm{DM}$, and chronic renal disease. The MAP and mean PI values were also significantly higher than in the control group.

The sensitivity of the combined method for detection of PE at any gestational age ( $<42$ weeks) was $41.2 \%$, with an FP rate of $10 \%$ and a negative predictive value of $96.2 \%$.

\section{Discussion}

In our study, the predictive performance using maternal characteristics, MAP, and uterine artery PI was inferior to the results obtaining using the FMF algorithm.

The high risk of developing PE in our sample was confirmed not only by the high incidence of PE (5.6\%), which was higher than expected in a low-risk population where the risk is usually 2 to $3 \%$ [12], but also by the augmented presence of risk factors. When comparing our results to those of the FMF case group, our sensitivity to this type of combined risk without biochemical markers was inferior. In group 2 ( $\mathrm{PE}<34$ weeks), our sensitivity was $71.4 \%$ with an FP rate of 10\%, whereas FMF sensitivity was $90 \%$. In group 3 ( $\mathrm{PE}<37$ weeks), our sensitivity was $50 \%$ vs. $72 \%$, and in group 4 ( $\mathrm{PE}<42$ weeks) our sensitivity was $41.2 \%$ vs. $57 \%$ [13]. It is worth noting that the FMF case group included around 30,000 patients, which means its statistical data have a higher impact.

The identification of patients who will develop early PE (before 34 weeks) is of great interest for further research since these cases have a greater disease severity. However, we cannot ignore the importance of identifying PE in preterm delivery ( $<37$ weeks). Late preterm infants between 34 and 37 weeks frequently require admission to the neonatal intensive care unit, which is not always available for most of our country's population. We would like to highlight that maternal repercussions can still be severe at this stage of pregnancy.

Furthermore, the recent disclosure of the results of the Aspirin for Evidence-Based PREeclampsia Prevention (ASPRE) study shows that early drug prophylaxis with ASA is effective in reducing the incidence of PE in early parturition, which increases the urgency to identify cases in this group $(<37$ weeks). The authors of this study used the FMF combined algorithm (maternal characteristics and biophysical and biochemical markers) with a sensitivity of $76 \%$ (FP 10\%) and a cut-off of 1/100 when detecting PE before 37 weeks (preterm) [14]. Patients at high risk for preterm PE that agreed to participate in the study were randomized into two groups: one group received ASA (150 mg/day) while the other received a placebo from the first trimester morphological evaluation until 36 weeks of gestation. The incidence of PE before 37 weeks was significantly lower in the group that received aspirin, with a reduction of $62 \%$ [14].

We believe that if obstetricians have a real possibility of implementing PE prophylaxis with ASA, they will have a greater interest in assessing the PE risk of each patient as part of prenatal routine practice. However, they must be aware of both the advantages and the limitations of the different screening methods. Also, despite having a reduced sensitivity without the use of biochemical markers, the prediction of PE using maternal characteristics associated with biophysical markers (MAP and uterine artery Doppler) is more accessible to our population since biochemical markers are not available in the Brazilian public health system. For this reason, the sensitivity is lower and may result in an increased number of women who use ASA without having any real risk of developing PE.

\section{Conflict of interest}

No potential conflict of interest relevant to this article was reported.

\section{References}

1. Lewis $G$. Reviewing maternal deaths to make pregnancy safer. Best Pract Res Clin Obstet Gynaecol 2008;22:447-63. 


\section{Obstetrics \& Gynecology Science}

Vol. 62, No. 6, 2019

2. ACOG Committee on Obstetric Practice; American College of Obstetricians and Gynecologists. ACOG practice bulletin. Diagnosis and management of preeclampsia and eclampsia. Number 33, January 2002. Int J Gynaecol Obstet 2002;77:67-75.

3. World Health Organization. Make every mother and child count. Geneva: World Health Organization; 2005.

4. Weindling AM. The confidential enquiry into maternal and child health (CEMACH). Arch Dis Child 2003;88:1034-7.

5. Martins AL. Maternal mortality among black women in Brazil. Cad Saude Publica 2006;22:2473-9.

6. Zanette E, Parpinelli MA, Surita FG, Costa ML, Haddad SM, Sousa MH, et al. Maternal near miss and death among women with severe hypertensive disorders: a Brazilian multicenter surveillance study. Reprod Health 2014;11:4.

7. Report of the national high blood pressure education program working group on high blood pressure in pregnancy. Am J Obstet Gynecol 2000;183:S1-22.

8. Ness RB, Roberts JM. Heterogeneous causes constituting the single syndrome of preeclampsia: a hypothesis and its implications. Am J Obstet Gynecol 1996;175:136570.

9. Bujold E, Roberge S, Lacasse $Y$, Bureau M, Audibert
F, Marcoux S, et al. Prevention of preeclampsia and intrauterine growth restriction with aspirin started in early pregnancy: a meta-analysis. Obstet Gynecol 2010;116:402-14.

10. Martin AM, Bindra R, Curcio P, Cicero S, Nicolaides KH. Screening for pre-eclampsia and fetal growth restriction by uterine artery Doppler at 11-14 weeks of gestation. Ultrasound Obstet Gynecol 2001;18:583-6.

11. Plasencia W, Maiz N, Bonino S, Kaihura C, Nicolaides $\mathrm{KH}$. Uterine artery Doppler at $11+0$ to $13+6$ weeks in the prediction of pre-eclampsia. Ultrasound Obstet Gynecol 2007;30:742-9.

12. Yu CK, Smith GC, Papageorghiou AT, Cacho AM, Nicolaides $\mathrm{KH}$; Fetal Medicine Foundation Second Trimester Screening Group. An integrated model for the prediction of preeclampsia using maternal factors and uterine artery Doppler velocimetry in unselected low-risk women. Am J Obstet Gynecol 2005;193:429-36.

13. Poon LC, Nicolaides KH. Early prediction of preeclampsia. Obstet Gynecol Int 2014;2014:297397.

14. Rolnik DL, Wright D, Poon LC, Syngelaki A, O'Gorman N, de Paco Matallana $C$, et al. ASPRE trial: performance of screening for preterm pre-eclampsia. Ultrasound Obstet Gynecol 2017;50:492-5. 